\title{
Tumor mutational burden in non-small cell lung cancer-the pathologist's point of view
}

\author{
Frédérique Penault-Llorca, Nina Radosevic-Robin \\ Department of pathology, Centre Jean Perrin, Clermont-Ferrand, France \\ Contributions: (I) Conception and design: F Penault-Llorca; (II) Administrative support: F Penault-Llorca; (III) Provision of study materials or \\ patients: None; (IV) Collection and assembly of data: None; (V) Data analysis and interpretation: None; (VI) Manuscript writing: All authors; (VII) \\ Final approval of manuscript: All authors. \\ Correspondence to: Penault-Llorca. Centre Jean Perrin, 58 Rue Montalembert, 63011 Clermont-Ferrand, France. \\ Email: frederique.penault-llorca@clermont.unicancer.fr.
}

\begin{abstract}
In non-small cell lung cancer (NSCLC), the pathologist has contributed to the development of personalized medicine from the determination of the right histological type to EGFR and ALK/ROS1 molecular screening for targeted therapies. With the development of immunotherapies, pathologists intervene forefront with programmed death-ligand 1 (PD-L1) immunohistochemical testing, companion test for pembrolizumab monotherapy, first line and complementary test to the other programmed cell death-1 (PD-1) PD-L1 inhibitors. Recently, tumor mutational burden has emerged as a promising tool to evaluate sensitivity to immunotherapy (IO). The pathologist has a crucial role in the setting of tumor mutational burden (TMB) testing for the selection and the preparation of the sample for high throughput molecular analysis, and in the first steps of the next-generation sequencing (NGS) workflow.
\end{abstract}

Keywords: Non-small cell lung cancer (NSCLC); biomarkers; immunotherapy (IO); tumor mutational load; preanalytical; quality assurance

Submitted Aug 13, 2018. Accepted for publication Sep 26, 2018.

doi: $10.21037 /$ tlcr.2018.09.26

View this article at: http://dx.doi.org/10.21037/tlcr.2018.09.26

The clinical breakthroughs of immunotherapy (IO) and personalized medicine have modified the survival and the quality of life of many cancer patients. These advances are possible through the development of predictive biomarkers of response to treatments. By using our own immune system to fight cancer, IO is also revolutionizing our concept of biomarkers (1). Thus, many emergent predictive biomarkers for cancer IO derive not only from the tumor cells, but also from their microenvironment, and from our innate capacity to develop an immune response, including major complex of histocompatibility and microbioma. The concept of a cancer immunogram combining different markers is emerging (2). The pathologist has a pivotal role in this quest for biomarkers in the setting of IO. Among the myriad of candidates, tumor mutational load or TMB is emerging as the new kid on the block (3).

\section{Pathologist and non-small cell lung cancer (NSCLC)}

\section{Diagnosis}

In lung cancer, the pathologist has contributed to the development of personalized medicine, starting with the determination of the right histologic subtype (problem solved by simple immunohistochemistry: TTF1, p40, CK5/6, neuroendocrine biomarkers) (4). This guide chemotherapy regimens for non-small cell and nonneuroendocrine carcinoma: i.e., gemzar, platinium salts and contraindication of anti-angiogenic therapy for squamous cell carcinoma (SCC); pemetrexed, platinium salts for nonsquamous. Moreover, histologic subtypes determination allows triage for molecular testing in NSCLC; SCC being excluded from molecular screening in the context of targeted therapies, so far (except for SCC developed non-smokers) (5). 


\section{Markers for IO}

The level programmed death-ligand 1 (PD-L1) expression detected by immunohistochemistry, on the surface of tumor cells, is so far, the unique validated biomarker for checkpoint inhibitors therapies anti programmed cell death-1 (PD-1) or PD-L1 in lung cancer (5-7). Although PD-L1 expression alone is often insufficient for patient selection in several tumor types. Recently, new insights have focused the important role of the tumor mutational load in this setting $(8,9)$. The tumor genome is a driver of anti-cancer immunity. Depending on the tumor mutational burden (TMB), the response to IO varies, suggesting that neoantigens generated by these mutations are crucial targets for $\mathrm{T}$ cells in cancer immunity (10). Today, TMB stands as one of the available tools to evaluate sensitivity to IO (3).

TMB accounts for the number of non-synonymous mutations per megabase. TMB is influenced by different factors including microsatellite instability, somatic mutations in POLE and POLD genes, exposition to tobacco, alkylating agents or sun, leading to a highly abnormal level of mutations in tumor DNA (11-14).

\section{Pathologist and TMB: genotype/phenotype associations?}

The first implication of the pathologist will be to identify tumors that can harbor a high mutation rate, such as tumors with a deficient mismatch repair (dMMR) pathway, or an hypermutator phenotype. Some pathological features such as poor differentiation, massive lymphocytic infiltration is more frequently observed in the context of dMMR, POLE exonuclease domain alterations (14-16) and are associated to high TMB (12). Yet, the reverse is not always true and for instance, melanoma, SCC, lung cancer frequently has a high TMB but are exceptionally dMMR $(<1 \%)$ or POLE deficient $(\sim 3 \%)(13,14,16,17)$.

The clinical history such as in colorectal cancer young age, right colon localization, is also informative for a potential dMMR phenotype, but no such clinical association exists in NSCLC where $\mathrm{dMMR}$ is found in $0.8 \%$ of lung adenocarcinoma (18). The quantification of tumor infiltrating lymphocytes or tumor-infiltrating lymphocytes (TILs) on haematoxylin and eosin (H\&E) slides has the potential to become a new tumor biomarker, being an easy approach to evaluate the immunogenicity of a tumor. The international TILs Guidelines provide standardization for a visual evaluation of $\mathrm{H} \& \mathrm{E}$ sections by pathologists in lung cancer (19). Their robustness has been demonstrated in international ring trials. Nevertheless, despite promising observations, linking the level of TILs and response to IO, more clinical validation is required to confirm TILs as strong predictive biomarkers for IO in lung cancer (20). In summary, the pathologist evaluating a tumor can give some valuable biomarkers for $\mathrm{IO}$ prediction such as $\mathrm{PD}-\mathrm{L} 1$ status, TILs count, dMMR status. However, those markers are neither exclusive nor $100 \%$ sensitive, but rather complementary of other biomarkers such as TMB, or composition of the microenvironment.

TMB is not standardized so far, but the techniques to explore it are complex and require the use of next generation sequencing. When implementing TMB measurement assays, it is important to consider factors that may affect the method workflow, the results of the test, and the interpretation of the data. Such factors include the type of specimen analyzed, sample quantity and quality, sequencing platform, genome coverage, bioinformatics pipeline, and the choice of the thresholds that determine a high TMB. The different techniques and their interpretation tools are detailed elsewhere in this special issue.

The pathologist has a crucial role in this setting for the selection and the preparation of the sample for high throughput molecular analysis, and in the first steps of the next-generation sequencing (NGS) workflow.

\section{Tissue samples}

Clinical samples for NSCLC diagnosis are heterogeneous. To ensure proper diagnosis and enough material for subsequent analysis, coordination between clinicians and pathologists is necessary. Different groups have published guidelines for clinicians concerning the number of samples required, to maximize the volume of tissue for diagnosis and subsequent analysis (21). At least five endobronchial/ transbronchial forceps biopsies \pm five additional, at least four EBUS aspirations per target, at least two percutaneous core needle biopsies with additional three to six are required for proper diagnosis and molecular testing. Cytoblocks have also recently being validated for IHC, FISH and molecular screening with small panels of genes. Surgical specimens are handled following codified procedures; collection of frozen material is optional. Three to five tumor sections $(5-10 \mathrm{~mm}$ thick) are sampled from the surgical specimen, after proper fixation (22).

As the amount of tissue is often limited, different strategies can be developed such as sample prioritization to 
spare tissue and allow the realization of all the analysis for accurate histological diagnosis (if needed) and, then, for the study of predictive biomarkers i.e., PD-L1 for all, and for non-SCC or SCC non-smokers, preservation of four to six blank sections for Alk, Ros1 by immunohistochemistry (and validation by fluorescent in situ hybridisation (FISH) under some circumstances) and molecular screening for mutations (EGFR at least, but usually KRAS, BRAF, HER2, ...) $(21,23)$. The management of the blocks has to be entrusted to qualified technicians.

Samples used for TMB assessment may be formalinfixed, paraffin-embedded (FFPE) tumor tissue, cytology samples or liquid biopsies. FFPE samples are the most commonly available for clinical testing, as the practise of systematic storage of frozen-specimens is not developed, especially for biopsies and in community centers. While some evidence exists for the feasibility of using cytology and fine-needle aspiration (FNA) samples for the analysis of small panels by NGS (24), these samples can yield variable amounts of DNA that may not always be sufficient for indepth molecular analysis $(25,26)$. In summary, more than $70 \%$ of patients have an advanced disease. Additional tests are frequently required. Thus, cytology or small biopsy samples with a small number of tumour cells might only allow diagnosis and classification of tumor type (i.e., adenocarcinoma). Then additional molecular tests may be compromised, especially large panel or whole exome sequencing (WES). New biopsies can be ordered by the multidisciplinary tumor board (MTB). Liquid biopsies represent an option for the future for TMB evaluation (27).

\section{Quality of the samples-preanalytical considerations}

FFPE samples present a risk for use with high-throughput genomic assays because preanalytical factors common to NGS testing can affect the sample integrity or amount of DNA that can be extracted $(26,28)$. An accurate molecular test requires a well-preserved specimen. The time of warm and cold ischemia should be as short as possible and can be monitored for a better traceability. Ischemia affects both protein and nucleic acids quality (29). The delay between tissue acquisition and fixation should be shorter than 30 minutes. It depends also upon the environmental temperature (23). The length of fixation depends on the size of the sample. Between 6-48 hours are recommended (12 optimal for a surgical specimen), in a sufficient quantity of fixative (30). Biopsies and cytology samples are fixed directly after the procedure, before the transfer to pathology. Cell blocks after centrifugation and formalin fixation are the favourite method for processing cytological specimens. They are handled like histological specimens. For surgical specimen a vacuum preservation can avoid delay to fixation, if the transfer to the pathology lab and the gross examination are expected to be superior to 30 minutes. Immediately after removal, the sample is conserved by sealing under vacuum in special plastic bags and placing it in a controlled environment $\left(4{ }^{\circ} \mathrm{C}\right)(21)$. The universally recommended fixative is $10 \%$ neutral buffered formalin (4\% formaldehyde).

Formalin fixation can affect sample integrity (31). FFPE tissues exhibit a high frequency of non-reproducible sequence alteration because of cross-linking cytosine nucleotides on DNA strands. Taq-DNA polymerase fails to recognize the cytosine and incorporates an adenine in the place of a guanosine, creating an artificial C-T or G-A mutation (32). The error rate is around $12 \%$ but those alterations are random and non-reproducible (33). FFPE slides and extracted DNA have been shown to be reliable for TMB assessment for 6 months and likely beyond (34).

\section{Sample selection for TMB: quantity}

The pathologist plays an important role in the selection of the best sample for molecular analysis. This sample should be as pure as possible in invasive neoplastic cells. The cellularity of the sample is evaluated in a standardized and reproducible manner, following guidelines (35). Cellularity is expressed as percentage of total cells/nuclei. This estimation is of outmost importance in determining success or failure of subsequent testing, by defining the lower limit of detection and a minimum percentage of neoplastic cells present can be applied (30\% for non NGS, $10 \%$ for NGS) $(21,23,24)$. A macrodissection allows a greater tumor DNA yield for assessment $(21,23,36)$. DNA quantity varies from 10-100 ng for gene panels and around $250 \mathrm{ng}$ for WES (36). It is critical to inspect the last section after the slides cut from the selected material delivered for molecular testing. If possible, avoid providing samples with extensive necrosis, pigmentation, huge inflammation. If not possible, due to sample limitation, this should be emphasized in the report, along with cellularity.

\section{Pathologist implication in the workflow}

Turnaround time is influenced by the time required for different steps in the NGS workflow. As TMB assessment 
is complex and long (run time and interpretation), there is increasing efforts to shorten the interval between the prescription of the molecular test by the oncologist and the time treatment is initiated, as this may influence prognosis. These upstream steps imply, surgeons, radiologists and pathologists $(21,36)$. The workflow steps under the responsibility of the pathologist encompass sample acquisition, pathology review and diagnosis (two to four days). Then the patient file is presented to the MTB, actionable mutation screening is prescribed (one week). If the sample centralized, le local pathologist has to batch and send to the central lab (variable length). As TMB assays are not routinely performed, there is a possibility of a second batch and send for TMB to a specialized lab (two to three weeks).

In lung cancer, before the development of NGS, the turnaround time for actionable mutation screening, i.e., EGFR and Alk, was approximatively five working days after the reception of the sample by the platform. A variable difficult to influence is the transfer duration between a local pathology department and a central molecular platform. During the last 10 years, with education and under the influence of scientific societies, the transfer times are reduced to three to five days in general (37-39). To improve the timing of molecular tests including TMB, we have some opportunities: (I) reflex testing of "routine" predictive markers by the pathologist after the diagnosis of a metastatic NSCLC; and (II) democratization of TMB assays to streamline diagnostic testing for TMB alongside other genomic markers, avoiding a second run.

\section{Quality assurance}

Quality assurance is requested at different steps of the TMB analysis. As previously mentioned this is a multidisciplinary shared concern. There is a need for all to be coordinated and well informed on the necessary approaches to achieve a reliable assay result. Molecular tumor boards have been created to guarantee global harmonization in tumor-sequencing practices (40). Concerning the pathologists, guidelines have been published to help the different structures (local or central) to devop standard operating procedures (SOPs) in order to harmonize practices and provide excellent quality nucleic acids (41). Efforts were developed to standardize the evaluation of tumor cellularity (35). It is important that these procedures be conducted in a standardized way. SOP as per ISO 15189 should be put in place from specimen identification to the final reports (36). Recent NGS guidelines underline the role of the pathology laboratory director in using an error-based approach that can detect potential sources of errors that could happen throughout the analytical process (41).

\section{Conclusions}

Validated biomarkers should accurately predict patient responses to IO. TMB assessment is emerging as a very promising factor. Using valuable lessons, learned from the implementation of targeted therapies in lung cancer, the pathologist has to play a key role in the development of $\mathrm{TMB}$ as a clinical diagnostic tool.

As the field evolves, reliable TMB assessments will be carried out in clinical practice, and included in the molecular workflow of lung cancer, as a reflex test. However, definitive recommendations may not be possible until more data are available from prospective clinical trials and external quality-control schemes to optimize the testing. Trained pathologists and technicians will provide high quality samples and efficient workflow to optimize advanced lung cancer patient's management.

\section{Acknowledgements}

None.

\section{Footnote}

Conflicts of Interest: Frédérique Penault-Llorca has received research grants and honoria from Astrazeneca, BMS, MSD and Roche. The other author has no conflicts of interest to declare.

\section{References}

1. Chen DS, Mellman I. Elements of cancer immunity and the cancer-immune set point. Nature 2017;541:321-30.

2. Blank CU, Haanen JB, Ribas A, et al. CANCER IMMUNOLOGY. The "cancer immunogram". Science 2016;352:658-60.

3. Rizvi NA, Hellmann MD, Snyder A, et al. Cancer immunology. Mutational landscape determines sensitivity to PD-1 blockade in non-small cell lung cancer. Science 2015;348:124-8.

4. Travis WD, Brambilla E, Noguchi M, et al. Diagnosis of lung cancer in small biopsies and cytology: implications of 
the 2011 International Association for the Study of Lung Cancer/American Thoracic Society/European Respiratory Society classification. Arch Pathol Lab Med;137:668-84.

5. Postmus PE, Kerr KM, Oudkerk M, et al; ESMO Guidelines Committee. Early and locally advanced nonsmall-cell lung cancer (NSCLC): ESMO Clinical Practice Guidelines for diagnosis, treatment and follow-up. Ann Oncol 2017;28:iv1-21.

6. Colwell J. Is PD-L1 Expression a Biomarker of Response? Cancer Discov 2015;5:1232.

7. Hersom M, Jorgensen JT. Companion and complementary diagnostics-focus on PD-L1 expression assays for PD-1/ PD-L1 checkpoint inhibitors in non-small cell lung cancer. Ther Drug Monit 2018;40:9-16.

8. Voong KR, Feliciano J, Becker D, et al. Beyond PD-L1 testing-emerging biomarkers for immunotherapy in nonsmall cell lung cancer. Ann Transl Med 2017;5:376.

9. Hellmann MD, Ciuleanu TE, Pluzanski A, et al. Nivolumab plus ipilimumab in lung cancer with a high tumor mutational burden. N Engl J Med 2018;378:2093-104.

10. Schumacher TN, Schreiber RD. Neoantigens in cancer immunotherapy. Science 2015;348:69-74.

11. Alexandrov LB, Nik-Zainal S, Wedge DC, et al. Signatures of mutational processes in human cancer. Nature 2013;500:415-21.

12. Chalmers ZR, Connelly CF, Fabrizio D, et al. Analysis of 100,000 human cancer genomes reveals the landscape of tumor mutational burden. Genome Med 2017;9:34.

13. Bonneville R, Krook MA, Kautto EA, et al. Landscape of microsatellite instability across 39 cancer types. In JCO Precis Oncol 2017;2017.

14. Campbell BB, Light N, Fabrizio D, et al. Comprehensive analysis of hypermutation in human cancer. Cell 2017;171:1042-56.e10.

15. Mensenkamp AR, Vogelaar IP, van Zelst-Stams WA, et al. Somatic mutations in MLH1 and MSH2 are a frequent cause of mismatch-repair deficiency in Lynch syndromelike tumors. Gastroenterology 2014;146:643-6.e8.

16. Song Z, Cheng G, Xu C, et al. Clinicopathological characteristics of POLE mutation in patients with nonsmall-cell lung cancer. Lung Cancer 2018;118:57-61.

17. Takamochi K, Takahashi F, Suehara Y, et al. DNA mismatch repair deficiency in surgically resected lung adenocarcinoma: microsatellite instability analysis using the Promega panel. Lung Cancer 2017;110:26-31.

18. Warth A, Körner S, Penzel R, et al. Microsatellite instability in pulmonary adenocarcinomas: a comprehensive study of 480 cases. Virchows Arch 2016;468:313-9.

19. Hendry S, Salgado R, Gevaert T, et al. Assessing TumorInfiltrating Lymphocytes in Solid Tumors: A Practical Review for Pathologists and Proposal for a Standardized Method from the International Immuno-Oncology Biomarkers Working Group: Part 2: TILs in Melanoma, Gastrointestinal Tract Carcinomas, Non-Small Cell Lung Carcinoma and Mesothelioma, Endometrial and Ovarian Carcinomas, Squamous Cell Carcinoma of the Head and Neck, Genitourinary Carcinomas, and Primary Brain Tumors. Adv Anat Pathol 2017;24:311-35.

20. Uryvaev A, Passhak M, Hershkovits D, et al. The role of tumor-infiltrating lymphocytes (TILs) as a predictive biomarker of response to anti-PD1 therapy in patients with metastatic non-small cell lung cancer or metastatic melanoma. Med Oncol 2018;35:25.

21. Dietel M, Bubendorf L, Dingemans AM, et al. Diagnostic procedures for non-small-cell lung cancer (NSCLC): recommendations of the European Expert Group. Thorax 2016;71:177-84.

22. Travis WD, Brambilla E, Burke AP, et al. editors. WHO Classification of Tumours of the Lung, Pleura, Thymus and Heart. Geneva: WHO Press; 2015.

23. Lindeman NI, Cagle PT, Aisner DL, et al. Updated Molecular Testing Guideline for the Selection of Lung Cancer Patients for Treatment With Targeted Tyrosine Kinase Inhibitors: Guideline From the College of American Pathologists, the International Association for the Study of Lung Cancer, and the Association for Molecular Pathology. J Thorac Oncol 2018;13:323-58.

24. DiBardino DM, Rawson DW, Saqi A, et al. Nextgeneration sequencing of non-small cell lung cancer using a customized, targeted sequencing panel: Emphasis on small biopsy and cytology. Cytojournal 2017;14:7.

25. Roy-Chowdhuri S, Chow CW, Kane MK, et al. Optimizing the DNA yield for molecular analysis from cytologic preparations. Cancer Cytopathol 2016;124:254-60.

26. Chen H, Luthra R, Goswami RS, et al. Analysis of preanalytic factors affecting the success of clinical nextgeneration sequencing of solid organ malignancies. Cancers (Basel) 2015;7:1699-715.

27. Koeppel F, Blanchard S, Jovelet C, et al. Whole exome sequencing for determination of tumor mutation load in liquid biopsy from advanced cancer patients. PLoS One 2017;12:e0188174.

28. Zhang P, Lehmann BD, Shyr Y, et al. The utilization 
of formalin fixed-paraffin-embedded specimens in high throughput genomic studies. In Int J Genomics 2017;2017:1926304.

29. Khoury T. Delay to Formalin Fixation (Cold Ischemia Time) Effect on Breast Cancer Molecules. Am J Clin Pathol 2018;149:275-92.

30. Kerr KM, Bubendorf L, Edelman MJ, et al. Panel Members; Panel Members. Second ESMO consensus conference on lung cancer: pathology and molecular biomarkers for non-small-cell lung cancer. Ann Oncol 2014;25:1681-90.

31. Einaga N, Yoshida A, Noda H, et al. Assessment of the quality of DNA from various formalin-fixed paraffinembedded (FFPE) tissues and the use of this DNA for next-generation sequencing (NGS) with no artifactual mutation. PLoS One 2017;12:e0176280.

32. Lamy A, Blanchard F, Le Pessot F, et al. Metastatic colorectal cancer KRAS genotyping in routine practice: results and pitfalls. Mod Pathol 2011;24:1090-100.

33. Prentice LM, Miller RR, Knaggs J, et al. Formalin fixation increases deamination mutation signature but should not lead to false positive mutations in clinical practice. PLoS One 2018;13:e0196434.

34. FDA. FoundationOne CDx: Summary of Safety and Effectiveness Data (SSED). Available online: https://www. accessdata.fda.gov/cdrh_docs/pdf17/P170019B.pdf

Cite this article as: Penault-Llorca F, Radosevic-Robin N. Tumor mutational burden in non-small cell lung cancerthe pathologist's point of view. Transl Lung Cancer Res 2018;7(6):716-721. doi: 10.21037/tlcr.2018.09.26
35. Lhermitte B, Egele C, Weingertner N, et al. Adequately defining tumor cell proportion in tissue samples for molecular testing improves interobserver reproducibility of its assessment. Virchows Arch 2017;470:21-7.

36. Cree IA, Deans Z, Ligtenberg MJ, et al. Guidance for laboratories performing molecular pathology for cancer patients. J Clin Pathol 2014;67:923-31.

37. Long É, Ilie $M$, Hofman V, et al. Role of the surgical pathologist for tissue management in oncology; Bull Cancer 2013;100:837-45.

38. Lièvre A, Artru P, Guiu M, et al. The KRAS mutation detection within the initial management of patients with metastatic colorectal cancer: a status report in France in 2011. Eur J Cancer 2013;49:2126-33.

39. Van Krieken JH, Rouleau E, Ligtenberg MJ, et al. RAS testing in metastatic colorectal cancer: advances in Europe. Virchows Arch 2016;468:383-96.

40. van der Velden DL, van Herpen CM, van Laarhoven HW, et al. Molecular tumor boards: current practice and future needs. Ann Oncol 2017;28:3070-5.

41. Jennings LJ, Arcila ME, Corless C, et al. Guidelines for validation of next-generation sequencing-based oncology panels: a joint consensus recommendation of the Association for Molecular Pathology and College of American Pathologists. J Mol Diagn 2017;19:341-65. 